\title{
Determinants of the implementation of hospital risk management: Case of the Provincial Hospital Center of El Jadida Morocco
}

\author{
Sakhr Ahizoune ${ }^{1 *}$, Rajaa Benkirane ${ }^{1,2}$, Mina El Hiyani ${ }^{1,3}$ and Asmaa Mdaghri Alaoui ${ }^{1,4,5}$ \\ ${ }^{1}$ Congenital anomalies research team, Center for medical biotechnology and therapeutic innovation, Faculty of Medicine and Pharmacy, University Mohammed V, \\ Rabat, Morocco \\ ${ }^{2}$ Public Health, National School of Public Health, Rabat, Morocco \\ ${ }^{3}$ Congenital anomalies research team, Center for medical biotechnology and therapeutic innovation, Faculty of Medicine and Pharmacy, University Mohammed V, \\ Rabat, Morocco \\ ${ }^{4}$ Dysmorphology unit, Pediatric 2 service Children's Hospital CHUIS RABAT \\ ${ }^{5} \mathrm{Head}$ of the Congenital anomalies research team, Center for medical biotechnology and therapeutic innovation, Faculty of Medicine and Pharmacy, University \\ Mohammed V Rabat, Morocco
}

\begin{abstract}
Introduction: Describe the determinants of the implementation of the Hospital Risk Management (HRM) approach at the Provincial Hospital Center (PHC) of El Jadida.

Methods: The study was conducted from July 2018 to December 2019 at the Provincial Hospital Center of El Jadida. It is a descriptive study, aims to describe the determinants of the implementation of the risk management approach at the PHC El Jadida through an interview with the heads of the HRM unit, a questionnaire for hospital managers and a questionnaire for professionals of the said PHC.

Results: Among the 338 participants in the study, more than half of them said that training and awareness are the main determinants for the implementation of the HRM approach in addition to the commitment of the administration. All of the managers interviewed agreed that the existence of the HRM unit is the starting point for any implementation of the HRM approach, while $87.1 \%$ stated that there was no HRM policy.

87.6\% of the professionals have not had any HRM training, while $97.6 \%$ of them have expressed their needs in terms of HRM training.

$85 \%$ of the professionals are unaware of the HRM procedure and the obligation to declare risks, which has impacted the implementation of this approach.

Discussion: The confrontation of our results allowed us to determine the determinants of the implementation of HRM, namely: (a) establishment of the HRM unit, (b) commitment of the administration, (c) awareness raising, (d) training, (e) design of an HRM policy, (f) institutionalization and regulation of the approach, (g) design of the HRM process.
\end{abstract}

Conclusion: Hospital risk management is essential for any hospital organization, enabling managers to make appropriate decisions.

\section{Introduction}

The report "To err is human: building a safer health system" highlighted the issue of hospital safety [1]. The latter illustrates the protection of human health against various risks [2]. So during this decade, hospital risk management has become inseparable from hospital safety and therefore constitutes a component of health policy [3]. Nowadays, hospital risk management has become an essential part of any hospital organization, because patient safety is a priority in medicine [4]. The implementation of hospital risk management is a key issue that the hospital of tomorrow cannot neglect [5]. Risk management is a decision-making tool as well as a means of guaranteeing the eternity of an organization [6]. The implementation of a risk management approach becomes essential, because the lack of a risk management system is the most important risk that the organization may face [6]. However, risk management is a major public health issue [7], in addition to being a topical subject illustrating a major concern of health authorities both nationally and internationally [8]. Hospital organizations manage the various risks informally, since it is an integral part of hospital activities and is part of an approach to improving the quality and safety of care in the hospital environment [9]. The implementation of a global risk management approach remains a major challenge for any organization, especially in the healthcare sector [6].

${ }^{\star}$ Correspondence to: Sakhr Ahizoune, Appartement 8, immeuble 6 RESIDENCE BAIT EL AZIZ, Avenue Tarik Ben Ziyad ilot 7, 12000 Temara, Morocco, Tel: +212674219555; Email: sakhrahizoune@gmail.com

Key words: risk management, hospital, HRM determinants

Received: February 11, 2021; Accepted: March 08, 2021; Published: March 12, 2021 
The notion of health safety and hospital risk management is a relatively recent concept in our Moroccan context, in terms of both strategic and regulatory orientations.

On the regulatory level, this concept emerged in 2008 following the implementation of the nine World Health Organization (WHO) recommendations [10], then the Ministry of Health $(\mathrm{MOH})$ implemented several measures and initiatives to ensure patient safety, notably through the adoption in 2002 of the WHO resolution, WHA55.18, on "Patient Safety", the development of several approaches to continuous improvement of quality and safety of care (implementation of the quality competition, certification of birthing centers, accreditation), participation in 2011 in the initiative led by the WHO EMRO Regional Office for Patient Safety in the countries of the region and the strengthening of the legal arsenal (Article 88 of the Internal Regulations of Hospitals, Framework Law 34-09 on the health system and the provision of care, etc.). These initiatives and measures were completed by the development and implementation of the Methodological Guide for Hospital Risk Management in Morocco [11] in 2016.

At the strategic level, the global report of the public consultation Intidarat Assiha published in 2013 revealed a more pressing demand from citizens, individuals and organizations on the theme of health monitoring and health security in health care institutions [12].

Despite all these efforts at the regulatory and strategic level, the operationalization of the risk management approach is incomplete and compartmentalized. This fact is confirmed by the results of the national organizational survey in 2005, which covered 49 hospitals and showed that quality assurance programs are present in 40 . In $8 \%$ of cases, annual action plans are written in $73.5 \%$ of cases, structures for the control of nosocomial infections exist in $69.4 \%$ of cases, procedures are written in $49 \%$ of cases, and monitoring through adverse event reporting forms is installed in $32.7 \%$ of the hospitals surveyed [13]. All these facts led the Ministry of Health to concretize the hospital risk management approach from 2016 by choosing two pilot sites, the PHC of El Jadida and the PHC of Tangier, with the aim of generalizing this approach to all Moroccan hospitals.

Our study aims to describe the determinants of the implementation of the hospital risk management approach at the PHC of El Jadida as it is a pilot site for the HRM approach.

\section{Material and study method}

\section{Study scheme}

This is a descriptive study. It aims to describe the determinants of the implementation of the risk management approach at the El Jadida Provincial Hospital Center and more precisely at the Mohammed V Hospital being a pilot site for this implementation.

\section{Duration of the study} 2019.

The study lasted eighteen months from July 2018 to December

\section{Target population}

The target population included all the health professionals of Mohammed V Hospital under the responsibility of El Jadida PHC, as the number of professionals is manageable $(n=338)$.

\section{Study site}

Mohammed V Hospital is a general hospital constituting the headquarters of the PHC El Jadida province, structured in five departments and two services and has a theoretical bed capacity of 460 beds with a functional bed capacity of 333 beds. The hospital has 338 health professionals including 233 nurses and health technicians, 63 doctors and 42 administrative professionals.

\section{Terms of reference}

The study has adopted Shortell's reference framework, which is based on four essential dimensions (or axes) that enable the development of a coherent, effective and sustainable approach [14].

\section{Data collection methods and tools}

In order to describe the determinants of the implementation of the hospital risk management (HRM) approach at the El Jadida PHC, interviews were conducted with the heads of the risk management unit, a questionnaire was distributed to hospital managers and finally a questionnaire was distributed to all professionals at the El Jadida PHC.

The questionnaires that dealt with the prerequisites and determinants of the implementation of the HRM approach were chosen as methods and tools for data collection from the hospital managers and professionals of the hospital, in addition to interviews with the heads of the hospital risk management unit. The results of the questionnaires and interviews, after their transcription, are presented in the form of tables and graphs with comments using the statistical analysis software SPSS and Excel (Figure 1).

\section{Ethical considerations}

The study respected ethical considerations, namely: having the permission and agreement of those in charge of the study environment; having the consent of the participants, after explicitly communicating the purpose and objectives of the research and the fate of the information given; openness, transparency and respect for the participants and guaranteeing their anonymity and confidentiality. As a result, we obtained the favorable opinion of the ethics committee.

\section{Results}

In order to describe the determinants of the implementation of the hospital risk management (HRM) approach at the El Jadida PHC, we divide the results according to the interviewed population, namely:

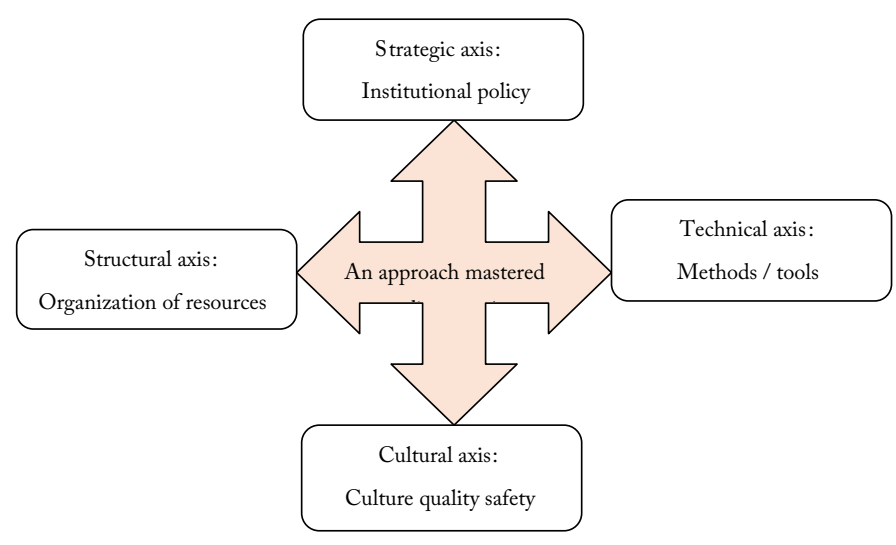

Figure 1. Shortell's four axes 
1. The members of the risk management unit;

2. Hospital managers;

3. The professionals of the HPC El Jadida.

About the results of the interviews with the members of the hospital risk management unit

The first interviews were carried out with the members of the hospital risk management unit implanted in the hospital in order to understand the context and determinants of the implementation of this approach as well as the tools used in the operationalization of the approach. All of the participants in the interviews confirmed that the implementation of the approach is the result of a new project conducted by the Ministry of Health with the aim of ensuring HRM and that the PHC of El Jadida is a pilot site, and affirmed that the starting point of the approach is illustrated by the commitment of the management. Half of the participants stated that awareness raising and training were the prerequisites for the implementation of this approach, while the rest of the interviewees stated that training was the only prerequisite to make this implementation a reality. All participants maintained that the steps in implementing the HRM approach consisted of establishing the context, identifying risks, analyzing risks, and addressing risks.

One quarter of the reported risks were care-related risks, $25 \%$ were nosocomial infections (NI), 25\% were blood exposure accidents (BEA), and the remaining $25 \%$ were equipment immobilization (EI).

Participants reported that $75 \%$ of the risk declarations were made by the risk declaration form, while $25 \%$ were made in written form.

All the participants reported that they organize periodic meetings to deal with the declarations issued by professionals, whereas they do not have a risk mapping neither a priori nor a posteriori. Finally, the participants emphasized the non-use of the hospital risk declaration application.

\section{Concerning the results of the questionnaire for the managers of the El Jadida PHC}

For the questionnaire intended for the managers of the El Jadida PHC belonging to the different hospital departments. The managers assured that $41.94 \%$ of the reported risks were care-related risks, while $25.81 \%$ were NI, $19.35 \%$ were BEA and $12.90 \%$ were EI.

Subsequently, $48.4 \%$ of managers stated that HRM is not institutionally way insured. $87.1 \%$ of participants stated that there is no HRM policy at the El Jadida PHC, and affirmed the existence of an HRM entity. $64.5 \%$ of managers assured the non-existence of an HRM action plan. $87.1 \%$ of participants felt that there is no improvement in the management of hospital risks. Finally, $77.4 \%$ of managers felt that there was no HRM assessment.

\section{For the results of the questionnaires for health professionals at the El Jadida PHC}

For HRM training, among 338 health professionals, $87.6 \%$ of them stated that they had no training in HRM, while only $12.4 \%$ had training in HRM as part of continuing education rather than initial training. 97.6\% of professionals require HRM training.

All participants faced risks that differed according to their work context. These risks were categorized by the participants as follows: $31.4 \%$ of care-related risks, $19.5 \%$ of NI, $19.2 \%$ of BEA, $16.6 \%$ of EI and $13.3 \%$ attributed to other risks.

\section{For risk reporting:}

- $85.8 \%$ of the participants were unaware of the risk reporting procedure;

- $84.9 \%$ of professionals were unaware of the obligation to declare risks;

- $96.7 \%$ of the participants declared the risks they had run.

As regards the supports of risk declaration:

- $48.5 \%$ of the professionals declared in writing,

- $33.7 \%$ reported orally,

- $14.5 \%$ per declaration form (3.3\% of responses missing).

$74.3 \%$ of professionals said they received feedback on their reports. Finally, all the professionals affirmed that they do not have risk mapping at the El Jadida PHC.

\section{Discussion}

Data collection from the risk management unit, hospital managers and professionals of the El Jadida PHC, allowed us to clarify the main determinants of the implementation of the HRM approach.

The implementation of the HRM approach at the El Jadida PHC was the result of a new Ministry of Health project designed to develop and improve the quality and safety of health care services in Moroccan public hospitals, This has been affirmed on the one hand by the results obtained and has been noted by other researchers in other contexts who agree that in the perspective of systemic promotion of risk management and assistance to institutions in this approach, their ministry provides a tool for implementing an HRM approach [3] and others attest that the implementation of the HRM approach is a major issue for any organization [6]. Some authors have revealed that risk management must find its rightful place in the hospital decision-making process [5]. Finally, the hospital horizon will progressively appear as a field also concerned by the concern for risk management [5], so the implementation of hospital risk management is a major issue that the hospital of tomorrow cannot neglect [5].

Interviewees emphasized the importance of management commitment to the implementation of the HRM approach, however, other authors argue that the implementation of an HRM approach within an institution requires a constant commitment from management [15].

Training and awareness-raising (information) were the main prerequisites for this implementation because the establishment of a safety culture in the hospital cannot escape these two determinants. This observation was confirmed with the interviewees and professionals participating in the study and was detected by other authors who point out that all professionals must be trained in prevention approaches and HRM tools and therefore training concerns managers, risk managers and teams involved in this approach [16]. Training is a pillar of change in any approach; it must be put into practice before any technical, equipment or work procedure changes, and more particularly organizational changes, and must be updated regularly [16]. Another researcher asserts that the training of health professionals is essential for the success of the HRM process [17]. The need for professionals in this area is increased, $97.6 \%$ in our study, whereas other researchers determine the need for HRM training at 91\% [17] and $85.71 \%$ [18]. However, in order for an HRM approach to be successful, a significant amount of training is necessary so that each professional can take 
ownership of the approach and a sustainable dynamic can be initiated [19].

Our results show that $87.6 \%$ of the professionals did not benefit from training in HRM, compared to other national studies which reveal $69 \%$ [17] and $65.33 \%$ [18] of the professionals had not received training in HRM, which has an impact on the one hand on the implementation of the HRM approach and on the other hand on the operationalization of the approach because $85.8 \%$ of the professionals were unaware of the procedure of risk declaration and $84.9 \%$ were unaware of the obligation of the declaration itself.

Raising awareness contributes in turn to the proper conduct of the HRM approach; $50 \%$ of the interviewees affirmed the usefulness of raising awareness in the implementation of the HRM approach, while other studies show that $77 \%$ were not made aware of risk management, which had a negative impact on the implementation of the approach [17]. A survey of 22 French healthcare institutions conducted by DHOS showed that more than half of the institutions surveyed had accompanied the implementation of the risk management program with a broad information and awareness campaign aimed at staff $[19,20]$, which explains the crucial role of information and awareness in the implementation of the HRM approach.

Risk reporting is critical to the success of the process because it is a crucial step in the HRM process and will determine the success of the implementation of the approach. It appears that the implementation of the compendium is the starting point for this approach and this compendium takes the form of a single declaration form [19]. Healthcare professionals declared the risks encountered in their services despite the minimal use of the official risk declaration form, although they declared orally or in writing to the hierarchy with consecutive rates of $33.7 \%$ and $48.5 \%$.

Half of the managers participating in the study stated that HRM is not institutionally insured, while Sghaiera, Hergona, Desroches foresee it as an integral part of any management system and as a cross-cutting activity of any organization [6].

The non-existence of the HRM policy, raised by $87.1 \%$ of managers, was evidence that the determinants and prerequisites for the implementation of the HRM approach have not been well operationalized. However, other authors have stated that the risk management policy must go beyond the simple declaration of risks that have occurred and move towards anticipatory action [19].

The majority of the hospital managers participating in the study confirmed the existence of the HRM unit, which is the cornerstone of the implementation of any HRM approach, despite the absence of an HRM policy. A risk management policy therefore requires steering and coordination structures [19].

All the participants revealed that they faced different risks depending on the location and department of their exercises, and therefore they face varying risks.

Finally, those in charge of the HRM unit, managers and professionals deplored the lack of a prior and a posteriori risk mapping.

\section{Conclusion}

To sum up, HRM is an indispensable approach that the hospital of tomorrow cannot deny in order to improve the quality and safety of the care provided. HRM is a necessary decision-making tool for any hospital manager, risk management must find the place it deserves in the hospital decision-making process [5]. The implementation of this approach implies the concretization of the determinants and prerequisites responsible for its success.

\section{References}

1. Kohn LT, Corrigan JM, Donaldson MS (2000) (Institute of medicine). In: To err is human: building a safer health system. Washington DC: National Academy Press, pp: 312 .

2. Tabuteau D (2002) Health safety. Edition Berger Levraut.

3. Farge-Broyart A, Rolland C (2005) National policy for risk management in health care institutions. Réanimation 14: 419-422.

4. Leveau P (2014) Safety of care in emergency medicine.

5. Belot C (2002) Risk management a new deal for hospital management: the example of the Stasbourg University Hospitals. Thesis ENSP.

6. Sghaiera W, Hergona E, Desroches A (2015) Global risk management. Clinical and Biological Transfusion 22: 158-167.

7. Pujola N (2015) Unplanned return to theater: A quality of care and risk management index? Orthopaedics \& Traumatology: Surgery \& Research 101: 399-403.

8. El Falaki A, Zerrouk L, Saad E (2020) Survey on risk management of medical devices in moroccan hospitals. IRBM News 41: 100266.

9. Department of Hospitalisation and Organisation of Care, DHOC. Circular DHOC/ E2/E4 no2004-176 - Recommendations for Developing and Implementing a Risk Management Program in Healthcare Facilities; 2004.

10. Circular $\mathrm{n}^{\circ} 97$ du 20 novembre 2008. Ministry of Health Morocco.

11. Methodological guide for hospital risk management in Morocco. 2016.

12. Overall report of the public consultation (Intidarat Assiha: Attentes en matière de santé), Ministry of Health Morocco. 2013. pp: 14.

13. Hospital and Ambulatory Care Branch (HACB), Enquête nationale organisationnelle. Maroc; 2005.

14. High Authority of Health. Patient Safety, a guide to using the pact team maturity matrix 2018.

15. Sophie Rouvillois. Initiate a global risk management policy in EPHAD. the example of the residence of the yze of naked bodies (35). ENSP. 2006. Rennes.

16. Lisbonaa A, Valerob M (2015) A priori risk analysis in practice. Cancer/Radiotherapy 19: 629-633.

17. Mars A (2017) Proposal for the implementation of a risk management approach associated with case care in the Regional Hospital Center of Agadir. ENSP. Morocco.

18. Amaati M (2014) Proposal for the implementation of a risk management approach in the hospital environment. Case of the Mohamed V provincial hospital center of Séfrou. ENSP. Morocco.

19. Dewaele L (2004) The contribution of a quality and health risk management approach to the dynamics of change in the local hospital. The example of the local hospital of BEAUGENCY. ENSP.

20. Broyart A (2001) Survey of risk management programs in healthcare facilities, 1st National Day on Health Risk Management in Healthcare Facilities, DHOC, pp: 6568.

Copyright: (C2021 Ahizoune S. This is an open-access article distributed under the terms of the Creative Commons Attribution License, which permits unrestricted use, distribution, and reproduction in any medium, provided the original author and source are credited. 